\title{
Analytical Solutions of Nonlinear Coupled Schrodinger-KdV Equation via Advance Exponential Expansion
}

\author{
Md. Mashiur Rahhman, Ayrin Aktar, Kamalesh Chandra Roy \\ Department of Mathematics, Begum Rokeya University, Rangpur, Bangladesh \\ Email address: \\ md.mashiur4182@gmail.com (Md. M. Rahhman)

\section{To cite this article:} \\ Md. Mashiur Rahhman, Ayrin Aktar, Kamalesh Chandra Roy. Analytical Solutions of Nonlinear Coupled Schrodinger-KdV Equation via \\ Advance Exponential Expansion. American Journal of Mathematical and Computer Modelling. Vol. 3, No. 3, 2018 , pp. 46-51. \\ doi: 10.11648/j.ajmcm.20180303.11
}

Received: November 21, 2018; Accepted: December 8, 2018; Published: February 18, 2019

\begin{abstract}
This research work is to represent an advance $\exp (-\Phi(\xi))$-expansion method with nonlinear ordinary differential equation for constructing interacting analytical solutions of nonlinear coupled physical models arising in science and engineering. It is capable of determining all branches of interacting analytical solutions simultaneously and this difficult to discriminate with numerical technique. To verify its computational potentiality, the coupled Schrodinger-KdV equation is considered. The obtained solutions in this work reveal that the method is a very effective and easily applicable of formulating the scattered exact traveling wave solutions of many nonlinear coupled wave equations. It is investigated the scattered wave solutions may be useful in understanding the behavior of physical structures in any varied instances, where the coupled Schrodinger-KdV equation is occurred.
\end{abstract}

Keywords: Coupled Schrodinger-KdV Equation, Solitary Wave Solution, Periodic Wave Solution, The Advance $\operatorname{Exp}(-\Phi(\xi))$-Expansion Method

\section{Introduction}

In this article, the advance $\exp (-\Phi(\xi))$-expansion method are mainly highlighted for finding more valuable explicit solutions of NLEEs. The valuable explicit form solution provides a means to determine the salient features in various science, technology and engineering applications. It can be serve as a basis for perfecting and testing computer algebraic software, such as Maple, Mathematica, MatLab etc for solving NLEEs. It is noted that several types of nonlinear partial differential equations (NPDEs) of physics, chemistry and biology hold unknown parameters and unknown functions. Analytical solutions allow researchers to design and perform experiments, by creating suitable natural situations, to determine these functions and parameters. There are several types of well-established methods that have been devoted to evaluate analytical solutions of NPDEs, such as the modified simple equation method $[1,2]$, the $\left(G^{\prime} / G\right)$ expansion method $[3,4]$, the tanh method $[5,6]$, the Homotopy perturbation technique [7], the homogeneous balance method [8,9], the Hirota method [10], the Expfunction method $[11,12]$, the $\exp (-\varphi(\xi))$-expansion method [13-18], the modified Kudryashov method [19], the generalized $\exp (-\varphi(\xi))$-expansion method [20, 21], and so on. Due to the effectiveness of mathematical approaches, the advance $\exp (-\Phi(\xi))$-expansion method may be easily applicable with the aid of symbolic computational software to find more general solitary and periodic wave solutions of NPDEs in mathematical physics and engineering. The main idea of this technique is to express the exact traveling wave solutions of NLEEs that satisfy the nonlinear ordinary differential equation (ODE) $\Phi^{\prime}(\xi)+\lambda \exp (\Phi(\xi))+\mu \exp (-\Phi(\xi))=0$, where $\lambda$ and $\mu$ real parameters. The advantage of this method over the other existing methods is that it provides some simple form exact traveling wave solutions to the nonlinear PDEs. Algebraic manipulations of this method is also much easier rather than the others existing methods.

However, many types of coupled NLEEs that appeared as model equations for describing the interacting wave 
phenomena in mathematical physics, chemistry and biology. For instance, the coupled Schrodinger-KdV equation are appeared as model equation to describe the interacting wave dynamics in Langmuir wave, dust-acoustic wave and electromagnetic wave in plasma physics, also appeared as a model equation to describe various types of wave phenomena in mathematical physics and so on. The existence and appearance of solitary waves in intricate physical issues apart from the model equations of mathematical physics must be analyzed with sufficient accuracy. Therefore, the work is to explore a study linking to the advance $\exp (-\Phi(\xi))$ expansion method for solving the coupled Schrodinger-KdV equation to demonstrate the effectiveness and truthfulness of this method.

\section{Analytical Solutions of Coupled Schrodinger-KdV Equation via the Advance $\exp (-\Phi(\xi))$-Expansion Method}

Let us consider the following coupled Schrodinger $-\mathrm{KdV}$ equation as

$$
\left.\begin{array}{l}
i u_{t}=u_{x x}+u v \\
v_{t}+6 v v_{x}+v_{x x x}=\left(|u|^{2}\right)_{x}
\end{array}\right\}
$$

Here $u(x, t)$ present the complex function while $v(x, t)$ present the real-valued function. The coupled Schrodinger $\mathrm{KdV}$ equation [22-24] appeared as model equation for describing various types of wave propagation such as Langmuir wave, dust-acoustic wave and electromagnetic waves in plasma physics. From the article [22-24], it is observed that some of the traveling wave solutions have been analyzed using different methods. In this article, the advance $\exp (-\Phi(\xi))$-expansion method is employed for finding more contented explicit from solutions to the coupled Schrodinger $-\mathrm{KdV}$ equation.

Besides, it is well known that the exp (- $\Phi(\xi))$-expansion method [13-17] has been employed to look into exact solutions of the nonlinear evolution equations, wherein the nonlinear $\operatorname{ODE} \quad \Phi^{\prime}(\xi)=\exp (-\Phi(\xi))+\mu \exp (\Phi(\xi))+\lambda \quad$; $\lambda, \mu \in \mathfrak{R}$ provides only a few traveling wave solutions to the nonlinear NLEEs. Therefore, in order to get more traveling wave solutions and to understand the inner structure apparently of the nonlinear physical phenomena, in this article we choose the following ODE as auxiliary equation:

$$
\Phi^{\prime}(\xi)+\lambda \exp (\Phi(\xi))+\mu \exp (-\Phi(\xi))=0, \lambda, \mu \in \Re .
$$

It is notable that eq. (4) has the following six kinds of general solutions as follows [18]:

$$
\begin{aligned}
& \Phi(\xi)=-\ln \left(\sqrt{\frac{\lambda}{\mu}} \tan \left[\sqrt{\lambda \mu}\left(\xi+\xi_{0}\right)\right]\right), \lambda \mu>0 \\
& \Phi(\xi)=-\ln \left(-\sqrt{\frac{\lambda}{\mu}} \cot \left[\sqrt{\lambda \mu}\left(\xi+\xi_{0}\right)\right]\right), \lambda \mu>0 \\
& \Phi(\xi)=-\ln \left(\sqrt{\frac{\lambda}{-\mu}} \tanh \left[\sqrt{-\lambda \mu}\left(\xi+\xi_{0}\right)\right]\right), \lambda \mu<0 \\
& \Phi(\xi)=-\ln \left(\sqrt{\frac{\lambda}{-\mu}} \operatorname{coth}\left[\sqrt{-\lambda \mu}\left(\xi+\xi_{0}\right)\right]\right), \lambda \mu<0 \\
& \Phi(\xi)=-\ln \left(-\frac{1}{\mu\left(\xi+\xi_{0}\right)}\right), \lambda=0, \mu>0, \\
& \Phi(\xi)=-\ln \left(\lambda\left(\xi+\xi_{0}\right)\right), \lambda \in \Re, \mu=0,
\end{aligned}
$$

where $\xi_{0}$ is the integrating constant and $\lambda \mu>0$ or $\lambda \mu<0$ depends on sign of $\lambda$.

Now, one can consider following travelling wave transform:

$u(x, t)=e^{i \omega} U(\xi), v(x, t)=v(\xi), \quad \omega=\alpha x+\beta t, \quad \xi=x+V t$,

where $\alpha, \beta$ and $V$ are constants. By substituting eq. (4) into eq. (1), one obtains that $V=2 \alpha$ and $U, v$ satisfy the following coupled nonlinear ordinary differential equations:

$$
\left.\begin{array}{l}
U^{\prime \prime}+\left(\beta-\alpha^{2}\right) U+U v=0 \\
2 \alpha v^{\prime}+6 v v^{\prime}+v^{\prime \prime \prime}-\left(U^{2}\right)^{\prime}=0
\end{array}\right\}
$$

where primes denotes the differentiation with regards to $\xi$. The pole of the coupled Eq. (5) are $N=2, M=2$. Therefore, the advance $\exp (-\varphi(\xi))$-expansion method allows us to use the solution in the following form:

$$
\left.\begin{array}{c}
U(\xi)=a_{0}+a_{1} \exp (-\varphi(\xi))+a_{2}(\exp (-\varphi(\xi)))^{2}, a_{2} \neq 0 \\
v(\xi)=b_{0}+b_{1} \exp (-\varphi(\xi))+b_{2}(\exp (-\varphi(\xi)))^{2}, b_{2} \neq 0
\end{array}\right\}
$$

where $a_{0}, a_{1}, a_{2}, b_{0}, b_{1}$ and $b_{2}$ are unknowns constants to be determined. By substituting eq. (6) in the eq. (5) and collecting all terms with same power of the coefficient of $e^{-\varphi(\xi)}$ together, we obtain a system of algebraic equations. The system of algebraic equations is ignored for convenience. Solving the obtaining system of algebraic equations, we obtain the following set of solutions:

Set 1: $\begin{gathered}\left\{\alpha=-3 b_{0}-8 \lambda \mu, \beta=-b_{0}+9 b_{0}^{2}+48 b_{0} \mu \lambda+64 \lambda^{2} \mu^{2}-6 \lambda \mu,\right. \\ \left.a_{0}=-4 \lambda \mu, a_{1}=0, a_{2}=-12 \mu^{2}, b_{0}=b_{0}, b_{1}=0, b_{2}=-6 \mu^{2}\right\}\end{gathered}$ 


$$
\text { Set 2: } \begin{array}{r}
\left\{\alpha=-3 b_{0}-16 \lambda \mu, \beta=-b_{0}+9 b_{0}^{2}+96 b_{0} \mu \lambda+256 \lambda^{2} \mu^{2}-2 \lambda \mu,\right. \\
\left.a_{0}=-12 \lambda \mu, a_{1}=0, a_{2}=-12 \mu^{2}, b_{0}=b_{0}, b_{1}=0, b_{2}=-6 \mu^{2}\right\}
\end{array}
$$

According to set 1 and set 2 and general solution of (3), the travelling wave solutions of the coupled Schrodinger-KdV equation (1) are obtained in the following form:

$$
\left.\begin{array}{c}
\left.u_{11}(x, t)=e^{i(\alpha x+\beta t)}\left\{-4 \lambda \mu-12 \lambda \mu \tan ^{2}\left(\sqrt{\lambda \mu}\left(x+2 \alpha t+\xi_{0}\right)\right)\right\}, \lambda \mu>0\right\} \\
v_{11}(x, t)=b_{0}-6 \lambda \mu \tan ^{2}\left(\sqrt{\lambda \mu}\left(x+2 \alpha t+\xi_{0}\right)\right), \lambda \mu>0 \\
\left.u_{12}(x, t)=e^{i(\alpha x+\beta t)}\left\{-4 \lambda \mu-12 \lambda \mu \cot ^{2}\left(\sqrt{\lambda \mu}\left(x+2 \alpha t+\xi_{0}\right)\right)\right\}, \lambda \mu>0\right\} \\
v_{12}(x, t)=b_{0}-6 \lambda \mu \cot ^{2}\left(\sqrt{\lambda \mu}\left(x+2 \alpha t+\xi_{0}\right)\right), \lambda \mu>0
\end{array}\right\}
$$

From the solutions (9) to (17), it is observed that the method according to subsidiary equation (2) is also given more valuable explicit form solutions to the coupled Schrodinger-KdV equation. These solutions would be more helpful to describe the interacting wave propagation of various physical phenomena in any varied natural instances. The obtaining solutions are also gives various types of solitary and periodic wave solutions according to the variation of the free parameters. Some of the important solitary and periodic wave solutions are presented in graphically. Since $u(x, t)$ is a complex function, the wave propagation in any natural varied instances are characterized by $|u(x, t)|$. Solution $u_{11}(x, t)$ present the periodic wave solutions in positive direction while solution $v_{11}(x, t)$ present the periodic wave solutions in negative direction corresponding to the values $\lambda=0.5, \mu=0.5, \xi_{0}=0$ and 
$b_{0}=-5 / 6$, which are shown in Figure 1. Solution $u_{12}(x, t)$ present the singular soliton type periodic wave solutions in positive direction while solution $v_{12}(x, t)$ present the singular soliton periodic wave solutions in negative direction corresponding to the values $\lambda=0.5, \mu=0.5, \xi_{0}=0$ and $b_{0}=-0.5$, which are shown in Figure 2. Solution $u_{13}(x, t)$ present the solitary wave solution of topological singular kink type while solution $v_{13}(x, t)$ present the solitary wave solution of non-topological bell type corresponds to the fixed values $\lambda=-0.5, \mu=0.5, \xi_{0}=0$ and $b_{0}=5 / 6$, which are shown in Figure 3. Solution $u_{14}(x, t)$ present the singular soliton type solitary wave in positive direction while $v_{14}(x, t)$ present the singular soliton type solitary wave in negative direction. The figures of the singular soliton type solitary waves are ignored for convenience. Solutions $u_{23}(x, t)$ and $u_{23}(x, t)$ are both presented the solitary wave solutions of non-topological bell type corresponds to the fixed values $\lambda=-0.5, \mu=0.5, \xi_{0}=0$ and $b_{0}=5 / 6$, which are shown in Figure 4. The others solutions are also presented various type of traveling wave solutions according to the variation of the parameters, which are ignored for convenience.

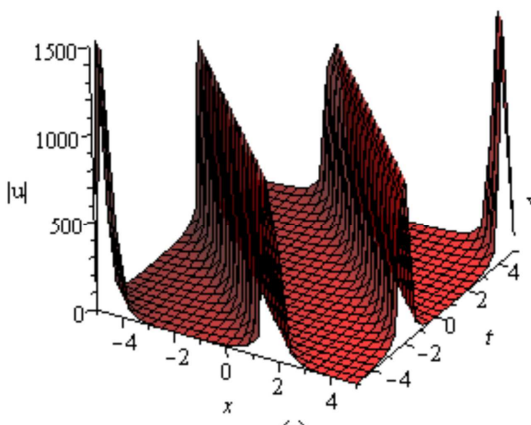

(a)

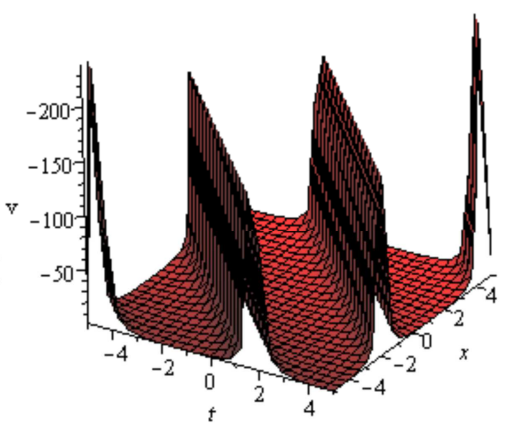

(b)

Figure 1. Shape of exact periodic traveling wave solutions of $(a)\left|u_{11}(x, t)\right|$ and (b) $v_{11}(x, t)$ with $-5 \leq x, t \leq 5$.

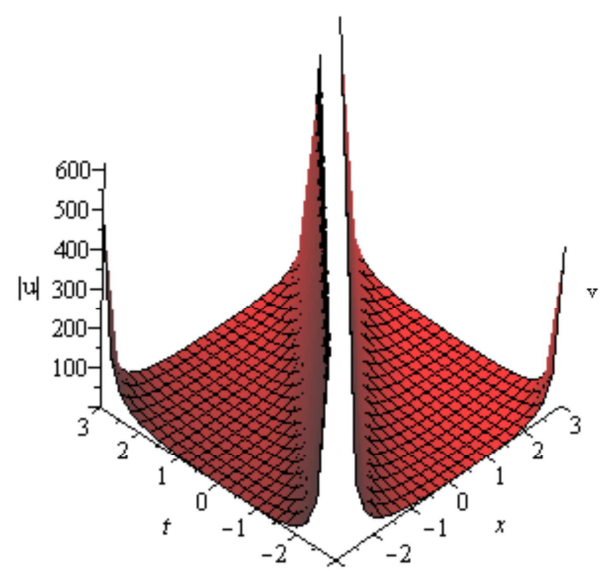

(a)

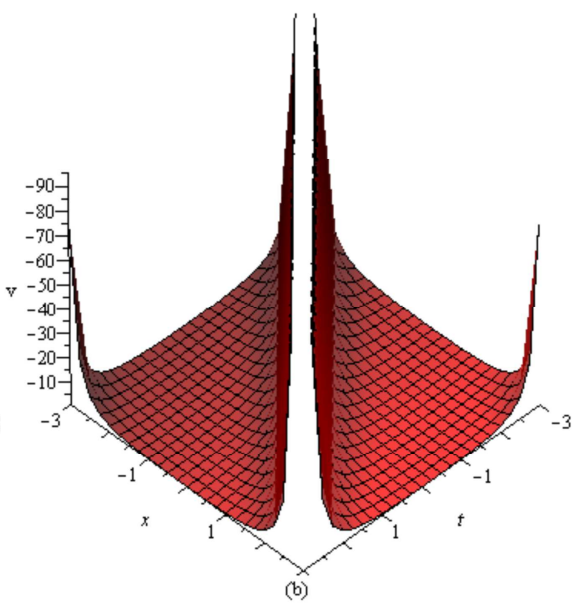

Figure 2. Shape of exact singular soliton type periodic traveling wave solutions of $(a)\left|u_{12}(x, t)\right|$ and (b) $v_{12}(x, t)$ with $-3 \leq x, t \leq 3$.

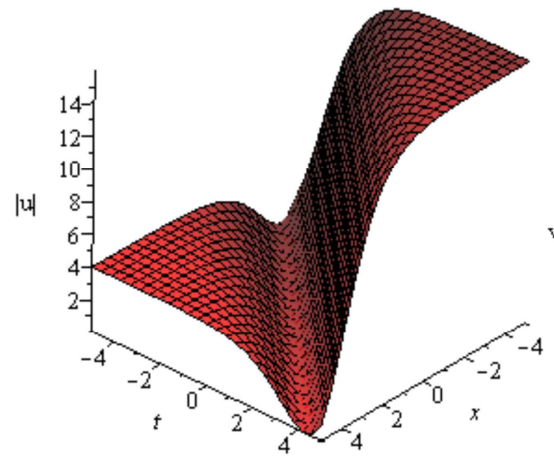

(a)

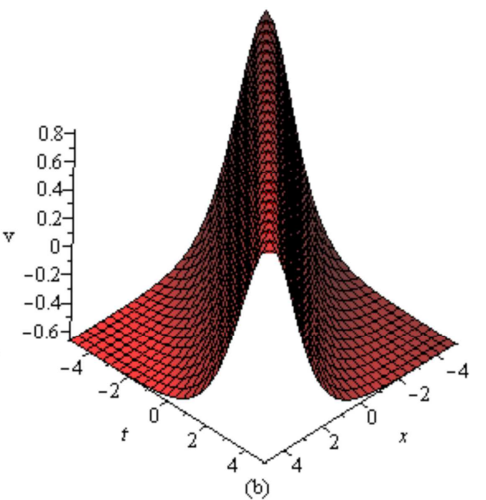

Figure 3. Shape of exact solitary wave solutions of (a) topological singular kink type for $\left|u_{13}(x, t)\right|$ and (b) non-topological bell type for $v_{13}(x, t)$ with 


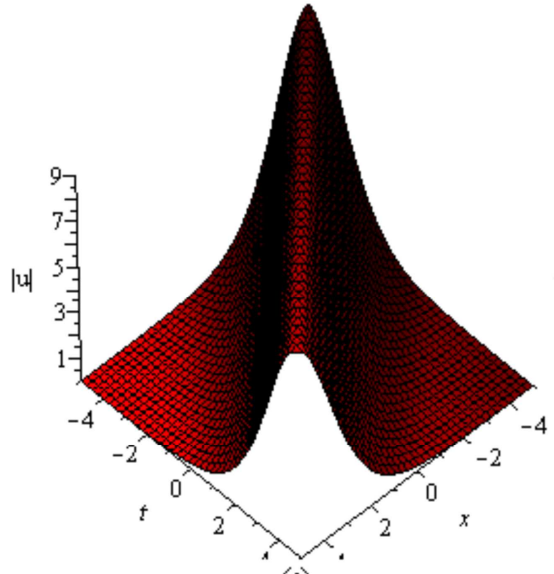

(a)

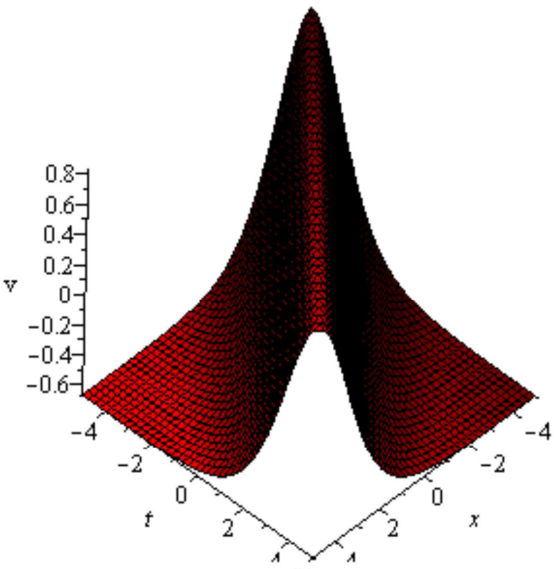

(b)

Figure 4. Shape of exact solitary wave solutions non-topological bell type of $(a)\left|u_{23}(x, t)\right|$ and (b) $v_{23}(x, t)$ with $-5 \leq x, t \leq 5$.

\section{Result and Discussions}

In this article, the ODE as in Eq. (2) has been considered as auxiliary equation and their solutions have been used. The main advantage of the introduced method is that it offers more simple form general exact traveling wave solutions with some free parameters. The exact solutions have its extensive importance to interpret the inner structures of the natural phenomena in mathematical physics, chemistry and biology. The explicit solutions represented various types of solitary wave solutions according to the variation of the physical parameters. In this article, some of types of solitary and periodic wave solutions are displayed graphically in Figures 1 to 4 . The advantage of this method is that sometimes gives solutions in disguised versions of known solutions that may be found by other methods. It is worth noted that the $\left(G^{\prime} / G\right)$-expansion method is special case of the extended $G^{\prime} / G$ )-expansion method. N. A. Kudryashov [25] have been shown that the $\left(G^{\prime} / G\right)$-expansion method is equivalent to the well known tanh-method. Besides, the solutions are achieved via the advance $\exp (-\Phi(\xi))$ expansion method with the auxiliary ODE $\Phi^{\prime}(\xi)+\lambda \exp (\Phi(\xi))+\mu \exp (-\Phi(\xi))=0$ while the $\left(G^{\prime} / G\right)-$ expansion method and the extended $\left(G^{\prime} / G\right)$-expansion method performed with others. It is concluded that some of our solutions may be coincided with already published results, if the parameters taken particular values which authenticate our solutions. Therefore, it can be decided that the method is powerful mathematical tool for easily solving nonlinear evolutions equations and all kinds of NLEEs may be solved through this method. It is also predicted form this investigations that the obtained results may be useful for better understanding interacting wave phenomena in any varied instance, where the considered coupled equations are applicable.

\section{Conclusions}

The advance $\exp (-\Phi(\xi))$-expansion method has been successfully employed to obtain generalized traveling wave solutions for describing the interacting wave phenomena in the vicinity of the coupled Schrodinger-KdV equation. The obtained solutions in this article are defined in the simple forms involving of hyperbolic functions, trigonometric functions and rational functions. It is found that the advance $\exp (-\Phi(\xi))$-expansion method changes the given difficult problems into simple problems which may be solved easily. Hence, this method may be more easily used to many others NLEEs arising in mathematical physics and engineering.

\section{References}

[1] Zayed EME. A note on the modified simple equation method applied to Sharma-Tasso-Olver equation. Appl. Math. Comput. 2011; 218 (7); 3962-3964.

[2] Zayed EME and Hoda Ibrahim SA. Modified simple equation method and its applications for some nonlinear evolution equations in mathematical physics. Int. J. Comput. Appli. 2013; 67 (6); 39-44.

[3] Wang ML, Li XZ, Zhang J. The $\left(G^{\prime} / G\right)$-expansion method and traveling wave solutions of nonlinear evolution equations in mathematical physics. Phys. Lett. A 2008; 372; 417-423.

[4] Hafez MG, Alam MN, Akbar MA, Exact traveling wave solutions to the Kelein_Gordon equation using the novel $\left(G^{\prime} / G\right)$-expansion method, Results in Phys. 2014; 4; 177-184.

[5] Wazwaz AM. The extended tanh-method for new compact and non-compact solutions for the KP-BBM and the ZK-BBM equations. Chaos, Solitons Fract. 2008; 38: 1505-1516.

[6] Malfliet W. The tanh method: A tool for solving certain classes of nonlinear evolution and wave equations. J. Comput. Appl. Math. 2004; 164: 529-541.

[7] Chun C, Sakthivel R. Homotopy perturbation technique for solving two point boundary value problems-comparison with other methods. Computer Phys. Commun. 2010; 181: 10211024.

[8] Zhao X, Tang D. A new note on a homogeneous balance 
method. Phys. Lett. A 2002; 297 (1-2); 59-67.

[9] Zhaosheng F. Comment on the extended applications of homogeneous balance method. Appl. Math. Comput. 2004; $158(2) ; 593-596$.

[10] Hirota R. Exact solution of the KdV equation for multiple collisions of solutions. Phys. Rev. Lett. 1971; 27; 1192-1194.

[11] Noor MA, Mohyud-Din ST, Waheed A. Exp-function method for travelling wave solutions of nonlinear evolution equations. Appl. Math. Comput. 2010; 216: 477-483.

[12] Noor MA, Mohyud-Din ST, Waheed A. Exp-function method for solving Kuramoto-Sivashinsky and Boussinesq equations. J. Appl. Math. Computing 2008; 29: 1-13.

[13] Akbar MA, Ali NHM. Solitary wave solutions of the fourth order Boussinesq equation through the $\exp (-\Phi(\eta))$-expansion method. SpringerPlus, 2014; 3-344. doi: 10.1186/2193-1801-3344.

[14] Hafez MG, Alam MN, Akbar MA. Traveling wave solutions for some important coupled nonlinear physical models via the coupled Higgs equation and the Maccari system. J. King Saud Uni.-Sci. in press, 2014. http://dx.doi.org/10.1016/j.jksus.2014.09.001

[15] Hafez MG, Kauser MA, Akter MT. Some new exact traveliing wave solutions of the cubic nonlinear Schrodinger equation using the $\exp (-\Phi(\xi))$-expansion method. Int. J. Sci. Eng. Tech. 2014; 3 (7); 848-851.

[16] Hafez MG, Kauser MA, Akter MT. Some new exact travelling wave solutions for the Zhiber-Shabat equation. British J. Math. Com. Sci. 2014; 4 (18); 2582-2593.
[17] Hafez, M. G.: Exact Solutions to the $(3+1)$-dimensional Coupled Klein-Gordon-Zakharov Equation Using Expexpansion Method. Alexandria Eng. J. 55, 1635 (2016).

[18] Hafez, M. G., Lu, D.: Traveling Wave Solutions for SpaceTime Fractional Nonlinear Evolution Equations. arXiv: 1512.00715 [math. AP] (2015).

[19] Ferdous F, Hafez M G. Oblique closed form solutions of some important fractional evolution equations via the modified Kudryashov method arising in physical problems. Journal of Ocean Engineering and Science 2018; 3: 244.

[20] Ferdous F, Hafez MG,. Ali MY. Obliquely propagating wave solutions to conformable time fractional extended ZakharovKuzetsov equation via the generalized $\exp (-(\xi))$-expansion method. SeMA 2018. https://doi.org/10.1007/s40324-0180164-2

[21] Ferdous F, Hafez MG. Nonlinear time fractional Korteweg-de Vries equations for interaction of wave phenomena in fluidfilled elastic tubes. Eur. Phys. J. Plus 2018; 133: 384.

[22] Lee J, Sakthivel R. Exact travelling wave solutions for some important nonlinear physical models. Pramana - J. Phys. 2013; 80: 757-769.

[23] Jabbari A, Kheiri H, Bekir A. Comput. Math. Appl. 2011; 62: 2177.

[24] Bahrami BS., Abdollahzadeh H, Berijani IM, Ganji DD, Abdollahzadeh M. Pramana - J. Phys. 2011; 77: 263.

[25] Kudryashov NA. A note on the $\left(G^{\prime} / G\right)$-expansion method. Appl. Math. Comput. 2010; 217 (4): 1755-1758. 